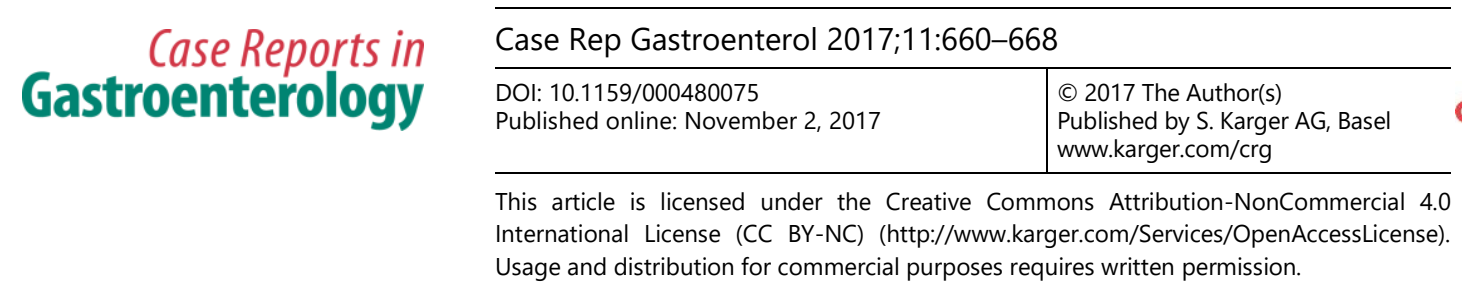

\title{
Gastric Adenocarcinoma with Dual Differentiation toward Neuroendocrine and $\alpha$-Fetoprotein-Producing Features: Report of 2 Cases
}

\author{
Shun lida ${ }^{a}$ Shinichi Ban ${ }^{a}$ Keizo Kato $^{b}$ Shinji Endo ${ }^{b}$ Ryota Matsuo ${ }^{c}$ \\ Kosuke Hirano $^{d}$ Takashi Okuyamad $^{d}$ Yukinori Yamagatad $^{d}$ \\ Yoshihiko Ueda ${ }^{a}$ \\ ${ }^{a}$ Department of Pathology, Dokkyo Medical University Koshigaya Hospital, \\ Koshigaya, Japan; 'bivision of Gastroenterology and Hepatology, Shinmatsudo Central \\ General Hospital, Matsudo, Japan; 'Division of Surgery, Shinmatsudo Central General \\ Hospital, Matsudo, Japan; dDepartment of Surgery, Dokkyo Medical University Koshigaya \\ Hospital, Koshigaya, Japan
}

\section{Keywords}

Gastric carcinoma N Neuroendocrine carcinoma $\cdot \alpha$-Fetoprotein-producing carcinoma $\cdot$ Dual differentiation

\begin{abstract}
Some gastric carcinomas show composite features of neuroendocrine carcinoma (NEC) and $\alpha$-fetoprotein (AFP)-producing carcinoma, which are very rare; only a few cases have been reported to date. We reviewed an additional 2 such cases of gastric carcinoma, which were both advanced aggressive tumors showing regional lymph node metastasis and distant metastasis. Both cases were accompanied by ordinary adenocarcinoma forms, implying that they had preceded the NEC and AFP-producing carcinoma components. A distinctive feature was the finding suggestive of dual differentiation of tumor cells to neuroendocrine and AFPproducing phenotypes, which was identified even in the metastatic tumor in the regional lymph node. Because both tumors predominantly showed poorly differentiated forms, the
\end{abstract}


lida et al.: Gastric Adenocarcinoma with Dual Differentiation toward Neuroendocrine and $\alpha$-Fetoprotein-Producing Features: Report of 2 Cases

final pathologic diagnosis must rely on the immunohistochemistry. Pathologists should always keep in mind the existence of such tumors for the correct diagnosis of some gastric carcinomas with specific phenotypes, especially in pathologic diagnosis on biopsy.

(C) 2017 The Author(s)

Published by S. Karger AG, Basel

\section{Introduction}

Some gastric carcinomas are dominantly comprised of tumor cells with specific phenotypes different from ordinary gastric adenocarcinoma, which include neuroendocrine carcinoma (NEC) [1] and $\alpha$-fetoprotein (AFP)-producing carcinoma [2]. Usually, NEC and AFPproducing carcinoma are distinct pathologic entities. However, a small number of cases showing composite features of NEC and AFP-producing carcinoma have been reported [3-6]. Recognition of the existence of such tumors is important because both NEC and AFPproducing carcinoma are characterized by a high incidence of metastasis and poor prognosis $[7,8]$, and appropriate immunohistochemistry is mandatory for their pathologic diagnosis.

We recently encountered an additional 2 gastric carcinoma cases with such composite features of NEC and AFP-producing carcinoma, in which dual differentiation of carcinoma cells toward NEC and AFP-producing carcinoma was suggested. We herein report these cases, considering some problems around the pathologic diagnosis of such neoplasms.

\section{Case Reports}

Case 1

A Japanese man in the fifth decade of life underwent gastroduodenal endoscopy, which revealed a large ulcerative lesion (type 3 [9]) occupying the anterior wall of the gastric antrum as well as involving part of the lesser and greater curvatures and the posterior wall. The lesion was diagnosed as adenocarcinoma on endoscopic biopsy, with regional and mediastinal lymph node metastasis and multiple liver metastases revealed by subsequent imaging studies. The patient died in a state of peritonitis carcinomatosa in spite of receiving 3 courses of chemotherapy (G-SOX); His death was followed by an autopsy. Before death, an elevated serum AFP level was identified, being $795.3 \mathrm{ng} / \mathrm{mL}$ (reference value $<10 \mathrm{ng} / \mathrm{mL}$ ).

On revaluation of endoscopic biopsies, the lesion included 2 histologic components, one being solid proliferation of large atypical cells with rounded and/or irregular vesicular nuclei showing a high nuclear-cytoplasmic (N/C) ratio (Fig. 1a), and the other being poorly differentiated adenocarcinoma comprised of atypical cells with PAS-positive relatively abundant cytoplasm and scattered formation of micro-lumens (Fig. 1b, c). Immunohistochemically, the former were diffusely positive for chromogranin A (Fig. 1d) and partly positive for AFP (Fig. 1e), whereas the latter were negative for both of them.

In the autopsied stomach, the tumor occupied the whole thickness of the gastric wall involving the serosa. Histologically, most of the tumor was comprised of invasive solid proliferation of large atypical cells with a high N/C ratio, which was consistent with one of the components observed in the biopsy tissue (Fig. 2a, b). However, in part of the ulcer bed side, some small carcinomatous nests were observed, in which carcinoma cells with relatively abundant pale eosinophilic cytoplasm proliferated, forming irregularly anastomosing trabeculae with intervening slit-like vessels, somewhat similar to a hepatoid appearance (Fig. $2 \mathrm{a}, \mathrm{c})$. Both components were immunoreactive to chromogranin $\mathrm{A}$, being consistent with 
lida et al.: Gastric Adenocarcinoma with Dual Differentiation toward Neuroendocrine and $\alpha$-Fetoprotein-Producing Features: Report of 2 Cases

NEC, but with stronger intensity in the component with a hetapoid appearance, part of which was also immunoreactive to AFP (Fig. 2d, e). The component of poorly differentiated adenocarcinoma observed in the biopsy tissue was not identified in the autopsy tissue. Metastatic carcinoma in both the regional and mediastinal lymph nodes showed the same histologic appearance, consistent with the NEC component mentioned above. Immunohistochemically, most of the metastatic carcinoma cells in the regional lymph node were positive for both chromogranin A and AFP, whereas those in the mediastinal lymph node were immunoreactive only to chromogranin $A$.

\section{Case 2}

A Japanese man in the sixth decade of life received distal gastrectomy for a tumor of the posterior wall of the lower gastric body, which had been diagnosed as adenocarcinoma on endoscopic biopsy. The resected tumor was a protruded mass measuring $2.4 \times 1.4 \times 1.5 \mathrm{~cm}$, surrounded by a geographic area showing a mixed flatly elevated and depressed appearance (type $1+0$-IIa + IIc [9]), measuring $5 \times 4 \times 1.5 \mathrm{~cm}$ as a whole (Fig. 3 ).

Histologically, the tumor invaded the proper muscle layer at the protruded portion, whereas it was limited to the mucosa and submucosa at the flatly elevated and depressed areas. The protruded mass was mainly comprised of compact proliferation of solid nests which consisted of large atypical cells with hyperchromatic round to oval nuclei showing a high N/C ratio (Fig. 4a, b). However, in the deep portion of the protruded mass, large polygonal neoplastic cells with pale eosinophilic cytoplasm proliferated, forming small nests and trabeculae with intervening thin vessels, showing a hepatoid pattern (Fig. 4a, c). The former compact solid nest portion was immunoreactive only to chromogranin A (Fig. 4d, e), and in the latter hepatoid area, some cells were immunoreactive to chromogranin A and/or AFP (Fig. 4d-f). Flatly elevated and depressed areas comprised moderately differentiated tubular adenocarcinoma showing anastomosing glands (Fig. 4g), in which scattered carcinoma cells were immunoreactive to chromogranin A (Fig. 4h), and AFP was positive for a limited focus (Fig. 4i). A regional lymph node with metastatic carcinoma was confirmed, which showed solid proliferation consistent with the NEC component and immunoreactive only to chromogranin A.

For both cases, immunohistochemical staining of the histologic sections was performed by Dako Autostainer Link 48 (Agilent Technologies, Santa Clara, CA, USA) according to the manufacturer's instructions, using the anti-chromogranin A monoclonal antibody (clone ZSA06; Nichirei, Tokyo, Japan) and the anti-AFP polyclonal antibody (Dako; Agilent Technologies).

\section{Discussion}

Gastric NECs and AFP-producing carcinomas are pathologically diagnosed as so when the tumors are dominantly composed of cells with each of such features. However, many of them reportedly included some components of ordinary adenocarcinoma, which has drown the concept that NECs and AFP-producing carcinomas would usually arise from pre-existing ordinary adenocarcinomas [1, 2, 10-12]. Some molecular studies also supported their evolution from ordinary adenocarcinomas. Akiyama et al. [12] confirmed that gastric hepatoid adenocarcinoma, a representative histologic type of AFP-producing carcinoma, and coexistent tubular adenocarcinoma had an identical origin by analyzing chromosome $\mathrm{X}$ inactivation, p53 mutation patterns, and/or the number of uniform LOH by microsatellite analysis. 
lida et al.: Gastric Adenocarcinoma with Dual Differentiation toward Neuroendocrine and $\alpha$-Fetoprotein-Producing Features: Report of 2 Cases

Nishikura et al. [1] suggested the occurrence of gastric NEC in the preceding adenocarcinoma components by analyzing p53 mutational status. The concept was strengthened by the fact that in some cases the AFP-producing carcinoma component emerged only in the metastatic tumors $[13,14]$.

The 2 cases presented here included both NEC and AFP-producing carcinoma components in each neoplasm. Such composite gastric carcinoma cases seem to be very rare [5], and to the best of our knowledge the reported cases in the English literature have been limited, all of which reportedly had ordinary adenocarcinoma components to some extent [3-6]. In particular, in the case reported by Okamoto et al. [4], the tubular adenocarcinoma component was limited in the mucosa in contrast to other components in the invasive areas, suggesting the origin of NEC and AFP-producing carcinoma components from the ordinary adenocarcinoma. The present 2 cases also had ordinary adenocarcinoma forms in addition to NEC and AFP-producing carcinoma ones, although in case 1 the autopsied lesion could not reveal them after chemotherapy. In case 2, most of the ordinary adenocarcinoma form with some positivity for chromogranin A and/or AFP was limited to the mucosa, which would imply the above-mentioned concept. The poorly differentiated histologic type of the ordinary adenocarcinoma component confirmed on the endoscopic biopsy material of case 1 needs to be noted because NECs and AFP-producing carcinomas usually originate from tubular and/or papillotubular adenocarcinomas [1,10-12].

A distinctive feature of case 1 was that chromogranin A-positive carcinoma cells and AFP-positive ones coexisted at the same lesion at least partly, including metastatic carcinoma cells in the regional lymph node, which suggests dual differentiation of the carcinoma cells to NEC and AFP-producing carcinoma. In case 2, the overlapping existence of chromogranin A-positive cells and AFP-positive cells was not as evident as in case 1. However, cells positive for chromogranin A and/or AFP were intermingled in the same area. These findings are different from the previously reported cases, in which the NEC component and the AFP-producing carcinoma component occupied different areas in the tumor [4-6], except 1 case suggesting the overlapping expression of chromogranin A and AFP [3]. The existence of carcinoma cells showing dual differentiation toward neuroendocrine and AFPproducing features is not surprising because the occurrence of a few neuroendocrine neoplastic cells in AFP-producing carcinomas has already been reported $[11,15]$. Besides, in the case reported by Okamoto et al. [4], carcinoma cells showing choriocarcinoma-like features were identified in the metastatic carcinoma in the lymph node, suggesting the potential of divergent differentiation of the composite gastric carcinomas.

The reported gastric composite carcinomas [3-6] and the present 2 cases were all advanced aggressive tumors showing regional lymph node metastasis and distant metastasis. It is of note that the most aggressive component in the tumor seems to be different in each case: In case 1, the metastatic carcinoma showed a composite feature, whereas in case 2 , the NEC component metastasized in the lymph node. In addition, in the previously reported cases, metastatic carcinoma was the AFP-producing carcinoma component in one case [6], whereas another case showed the NEC component with choriocarcinoma-like cells [4]. Besides, the latter case was somewhat complicated because the recurrent carcinoma after resection showed AFP-producing carcinoma features.

In case 1 , it seems to be plausible that only the ordinary adenocarcinoma component was sensitive to the oncostatic drugs. For predicting the outcome of patients and considering the possible development of drugs targeted at each component of the composite tumors in the future, it is desirable to recognize all of the tumor components pathologically. However, the histologic pattern is not always typical to NEC and/or AFP-producing carcinoma, as 
lida et al.: Gastric Adenocarcinoma with Dual Differentiation toward Neuroendocrine and $\alpha$-Fetoprotein-Producing Features: Report of 2 Cases

shown in the present 2 cases. Therefore, the application of immunohistochemistry should be considered if specific phenotypes of gastric carcinoma are suspected because of complicated histologic appearance with poorly differentiated morphology. Besides, biopsy diagnosis before treatment is much more problematic because sampling errors would fail to reveal the components of the tumor and/or specific phenotypes could not be recognized in small biopsy samples, as was seen in the present 2 cases and 1 previous report [4] in which diagnoses of merely adenocarcinoma were made. As for this point, one hint seems to be the endoscopic (macroscopic) shape of the tumors. The present case 2 and the 2 previously reported cases $[4,6]$ showed complicated macroscopic forms comprised of a polypoid/protruded mass and a depressed/ulcerative area, which would reflect the composite histologic architecture of the tumors (medullary growth of the NEC component formed the polypoid/protruded mass). Therefore, if a gastric tumor reveals a complicated endoscopic finding, multiple biopsy samples should be obtained from various areas of the tumor, and in that situation, pathologists should always consider the possibility of specific phenotypes of gastric carcinoma.

\section{Statement of Ethics}

The authors have no ethical conflicts to disclose.

\section{Disclosure Statement}

The authors have no conflicts of interest to disclose.

\section{References}

1 Nishikura K, Watanabe $\mathrm{H}$, Iwafuchi M, et al: Carcinogenesis of gastric endocrine cell carcinoma: analysis of histopathology and p53 gene alteration. Gastric Cancer 2003;6:203-209.

2 Kinjo T, Taniguchi H, Kushima R, et al: Histologic and immunohistochemical analyses of $\alpha$-fetoproteinproducing cancer of the stomach. Am J Surg Pathol 2012;36:56-65.

-3 Rassidakis GZ, Delladetsima JK, Letsos SP, et al: Hepatoid adenocarcinoma of the stomach with extensive neuroendocrine differentiation and a coexisting carcinoid tumour. Histopathology 1998;33:186-188.

4 Okamoto T, Ogasahara K, Fujiki M, et al: Primary coexistent neuroendocrine carcinoma, hepatoid adenocarcinoma, and tubular adenocarcinoma of the stomach with focal trophoblastic differentiation in metastatic lymph nodes. J Gastroenterol 2003;38:608-610.

-5 Suzuki A, Koide N, Kitazawa M, et al: Gastric composite tumor of alpha fetoprotein-producing carcinoma/hepatoid adenocarcinoma and endocrine carcinoma with reference to cellular phenotypes. Patholog Res Int 2012;2012:201375.

-6 Lipi L, Sachdev R, Gautam D, et al: Triple composite tumor of stomach: a rare combination of alpha fetoprotein positive hepatoid adenocarcinoma, tubular adenocarcinoma and large cell neuroendocrine carcinoma. Indian J Pathol Microbiol 2014;57:98-100.

-7 Yamaguchi T, Machida N, Morizane C, et al: Multicenter retrospective analysis of systemic chemotherapy for advanced neuroendocrine carcinoma of the digestive system. Cancer Sci 2014;105:1176-1181.

8 Inoue M, Sano T, Kuchiba A, et al: Long-term results of gastrectomy for alpha-fetoprotein-producing gastric cancer. Br J Surg 2010;97:1056-1061.

-9 Japanese Gastric Cancer Association: Japanese classification of gastric carcinoma: 3rd English edition. Gastric Cancer 2011;14:101-112.

10 Ishikura H, Kirimoto K, Shamoto M, et al: Hepatoid adenocarcinomas of the stomach. An analysis of seven cases. Cancer 1986;58:119-126.

11 Kashimura H, Shimoda T, Ikegami M: The phenotypic expressions of AFP producing gastric carcinoma (in Japanese). Nihon Shokakibyo Gakkai Zasshi 1995;92:751-760. 


\section{Case Reports in \\ Gastroenterology}

\begin{tabular}{l|l}
\hline Case Rep Gastroenterol 2017;11:660-668 \\
\hline DOI: 10.1159/000480075 & $\begin{array}{l}\text { @ 2017 The Author(s). Published by S. Karger AG, Basel } \\
\text { www.karger.com/crg }\end{array}$ \\
\hline
\end{tabular}

lida et al: Gastric Adenocarcinoma with Dual Differentiation toward Neuroendocrine and $\alpha$-Fetoprotein-Producing Features: Report of 2 Cases

12 Akiyama S, Tamura G, Endoh Y, et al: Histogenesis of hepatoid adenocarcinoma of the stomach: molecular evidence of identical origin with coexistent tubular adenocarcinoma. Int J Cancer 2003;106:510-515

13 Kodama T, Kameya T, Hirota T, et al: Production of alpha-fetoprotein, normal serum proteins, and human chorionic gonadotropin in stomach cancer: histologic and immunohistochemical analyses of 35 cases. Cancer 1981;48:1647-1655.

14 Kang GH, Kim YI: Alpha-fetoprotein-producing gastric carcinoma presenting focal hepatoid differentiation in metastatic lymph nodes. Virchows Arch 1998;432:85-87.

$\checkmark 15$ Nagai E, Ueyama T, Yao T, et al: Hepatoid adenocarcinoma of the stomach. A clinicopathologic and immunohistochemical analysis. Cancer 1993;72:1827-1835.
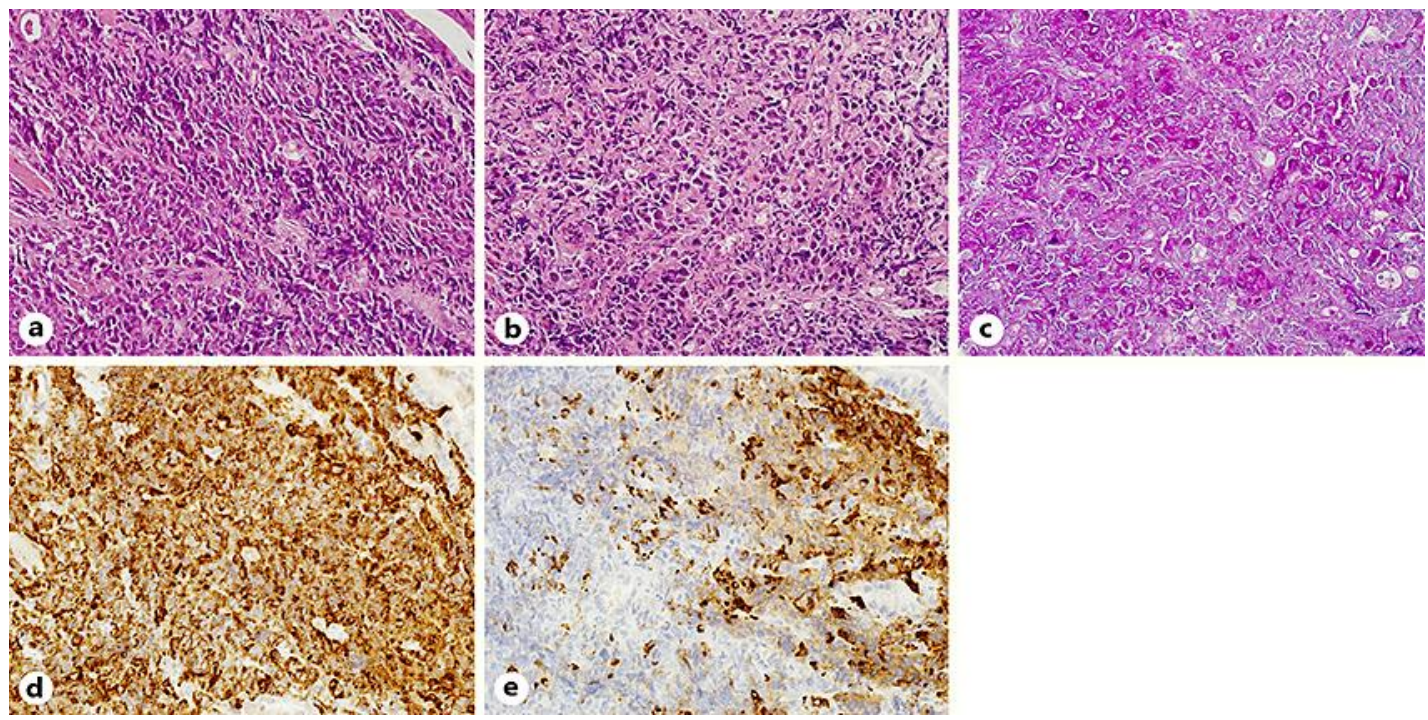

Fig. 1. Histologic findings of the endoscopic biopsy of the case 1 tumor including 2 components: solid proliferation of large atypical cells with rounded and/or irregular vesicular nuclei showing a high N/C ratio (a) and poorly differentiated adenocarcinoma comprised of atypical cells with PAS-positive relatively abundant cytoplasm and scattered formation of micro-lumens (b, c). Immunohistochemically, the former are diffusely positive for chromogranin A (d) and partly positive for $\alpha$-fetoprotein (e). 

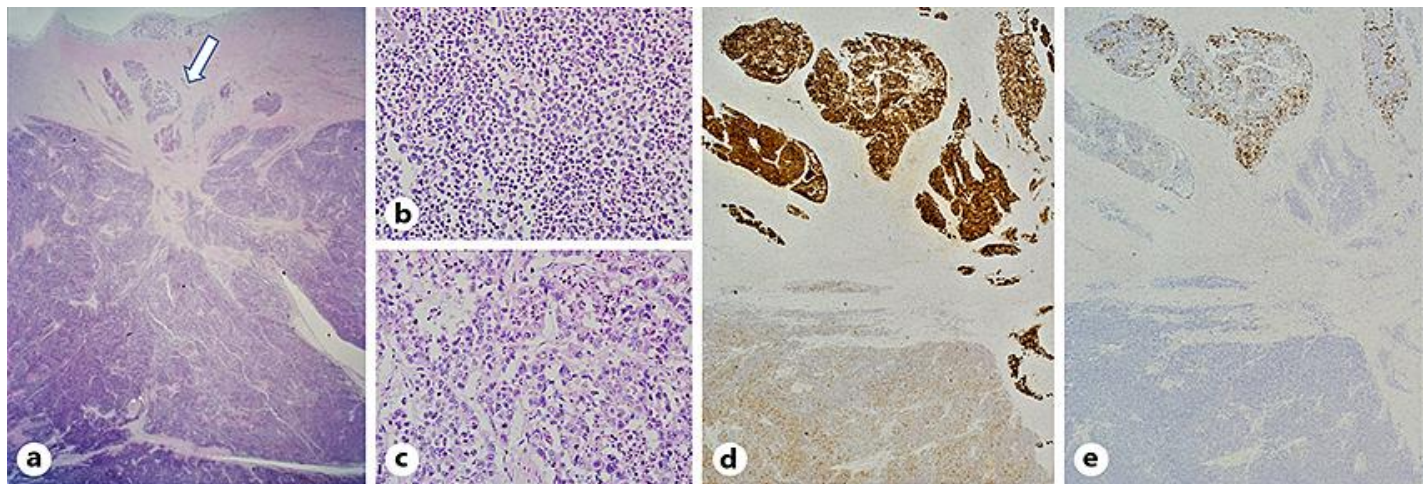

Fig. 2. Histologic findings of the case 1 tumor of the autopsied stomach. In low-power view (a), most of the tumor is comprised of invasive solid proliferation of large atypical cells with a high N/C ratio (b), whereas in part of the ulcer bed side (arrow in a) carcinoma cells with relatively abundant pale eosinophilic cytoplasm proliferate, forming irregularly anastomosing trabeculae with intervening slit-like vessels, somewhat similar to a hepatoid appearance (c). Immunohistochemically, both components are immunoreactive to chromogranin A (d) but with stronger intensity in the component with a hetapoid appearance (upper half of d), part of which is also immunoreactive to $\alpha$-fetoprotein (e).

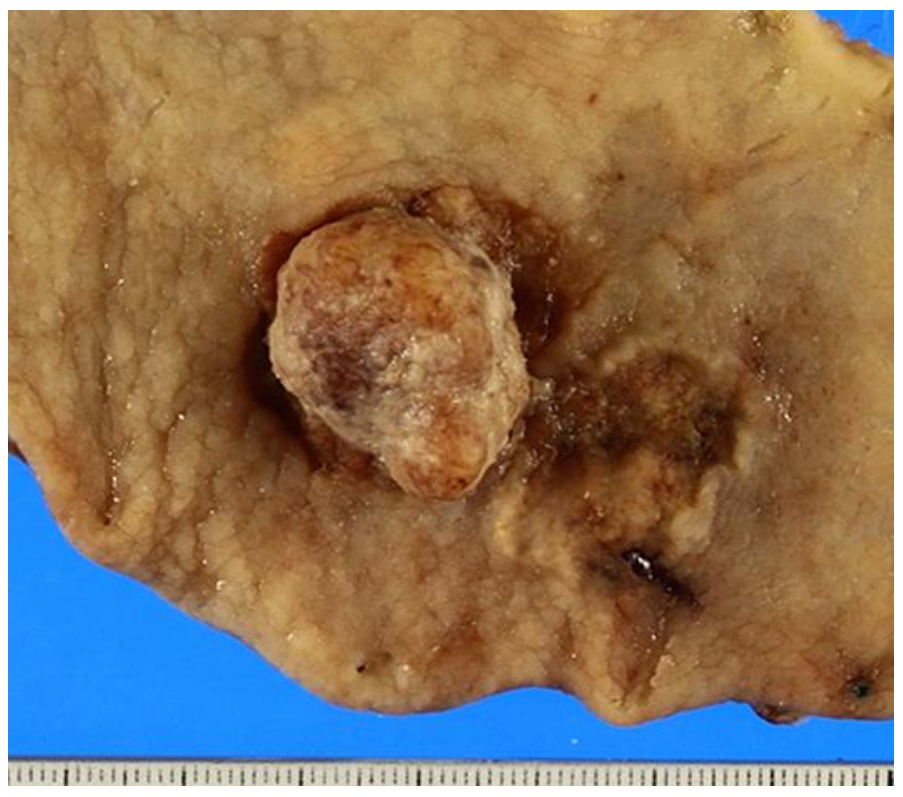

Fig. 3. Gross finding of the case 2 tumor in the resected stomach, comprised of a protruded mass measuring $2.4 \times 1.4 \times 1.5 \mathrm{~cm}$, surrounded by a geographic area showing a mixed flatly elevated and depressed appearance, measuring $5 \times 4 \times 1.5 \mathrm{~cm}$ as a whole. 


\section{Case Reports in Gastroenterology}
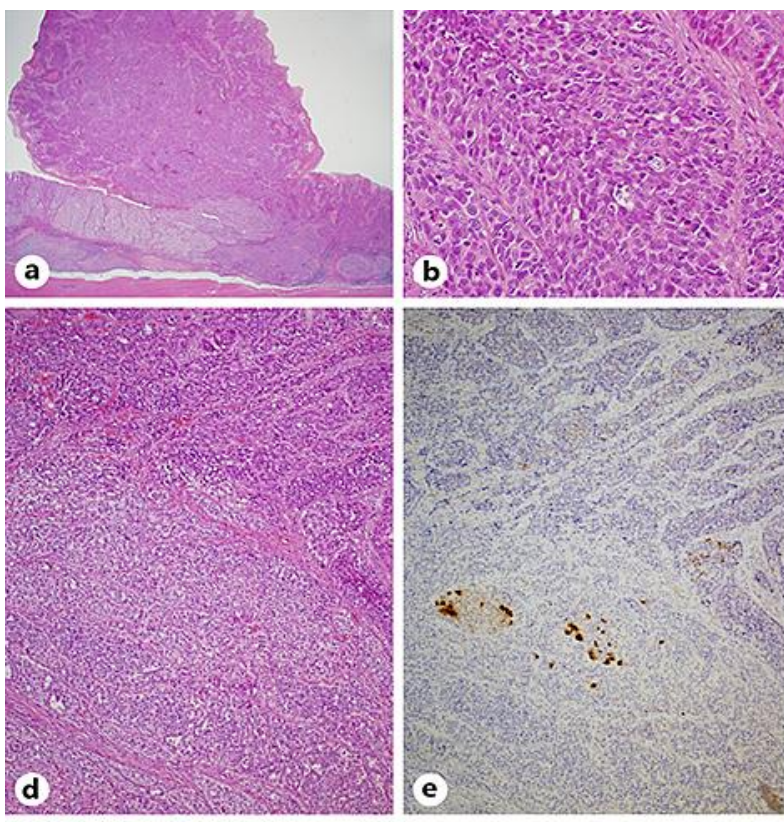
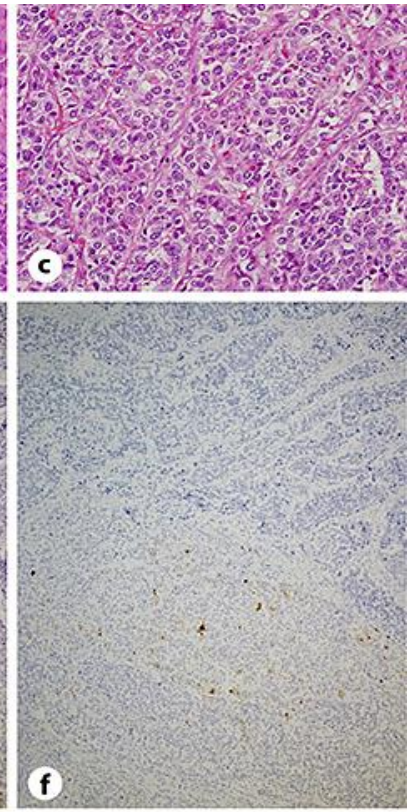
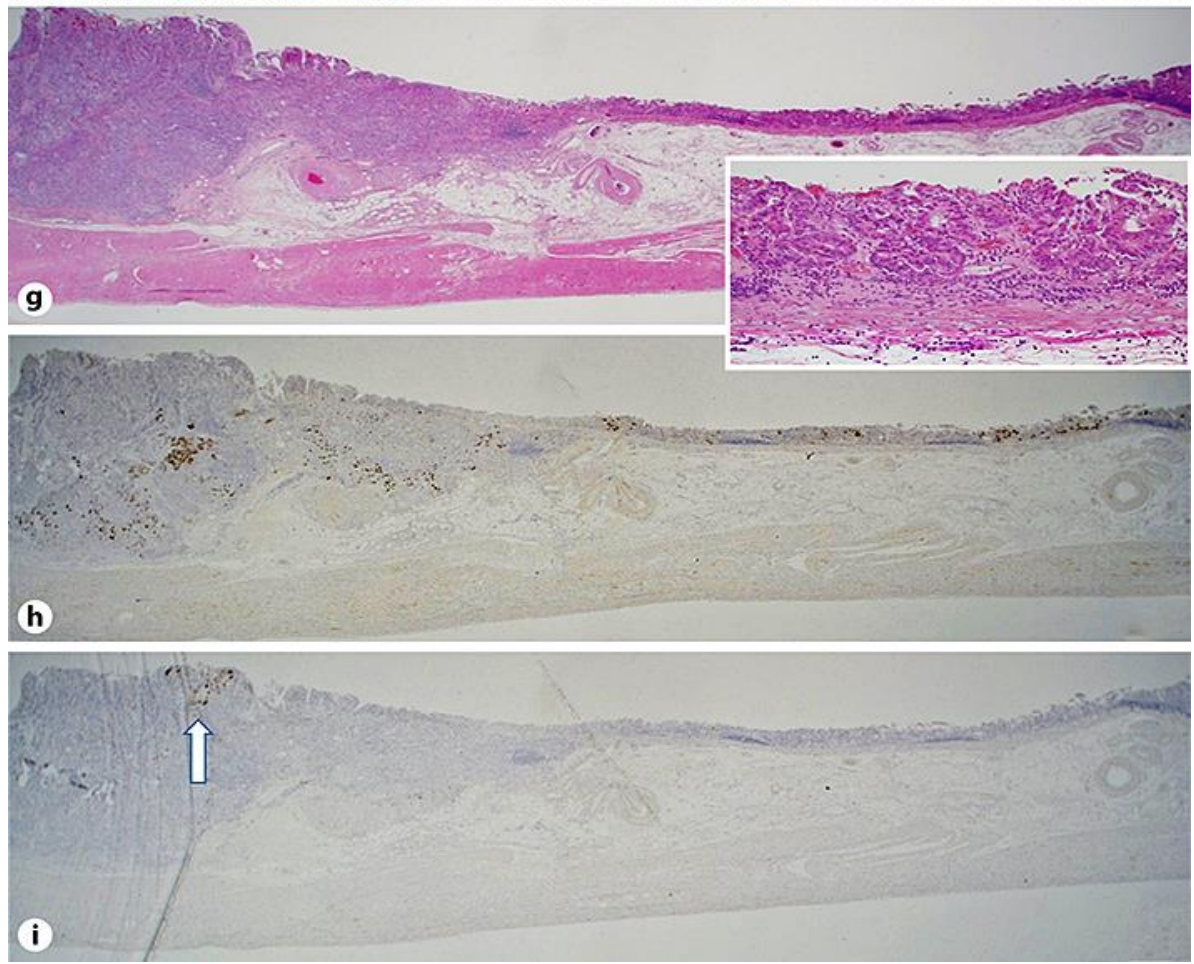


\section{Case Reports in Gastroenterology}

\begin{tabular}{l|l}
\hline Case Rep Gastroenterol 2017;11:660-668 \\
\hline DOI: 10.1159/000480075 & $\begin{array}{l}\text { @ 2017 The Author(s). Published by S. Karger AG, Basel } \\
\text { www.karger.com/crg }\end{array}$ \\
\hline
\end{tabular}

lida et al.: Gastric Adenocarcinoma with Dual Differentiation toward Neuroendocrine and $\alpha$-Fetoprotein-Producing Features: Report of 2 Cases

Fig. 4. Histologic findings of the case 2 tumor in the resected stomach. In low-power view of the protruded mass (a), the upper two-third dark portion is mainly comprised of compact proliferation of solid nests consisting of large atypical cells with hyperchromatic round to oval nuclei showing a high N/C ratio (b), whereas in the deep one-third clear portion, large polygonal neoplastic cells with pale eosinophilic cytoplasm proliferate, forming small nests and trabeculae with intervening thin vessels, showing a hepatoid pattern (c). In middle-power view of the boundary area of the 2 components (d), the compact solid nest portion (upper right half of $\mathbf{d}$ ) is immunoreactive only to chromogranin A (e), and some cells of the hepatoid portion (lower left half of d) are immunoreactive to chromogranin A and/or $\alpha$-fetoprotein (f). In low-power view of the flatly elevated and depressed area $(\mathbf{g})$, in which the tumor is limited to the submucosa (left half) or the mucosa (right half), moderately differentiated tubular adenocarcinoma showing anastomosing glands is observed (g, Insert). The tubular adenocarcinoma is immunoreactive to chromogranin A in a scattered manner (h) and to $\alpha$-fetoprotein in a limited focus (i, arrow). 